\title{
Ergodic Endomorphisms of Compact Abelian Groups ${ }^{\star}$
}

\author{
M. Shirvani and T. D. Rogers \\ Department of Mathematics, University of Alberta, Edmonton, Alberta Canada. T6G 2G1
}

\begin{abstract}
We show that for a surjective endomorphism of a compact abelian group ergodicity is equivalent to a condition which implies $r$-mixing for all $r \geqq 1$, and we characterize such maps algebraically. This is then used in proving the ergodicity of an extensive class of endomorphisms of the binary sequence space. As a simple corollary it is found that one-dimensional linear cellular automata and the accumulator automata are $r$-mixing for all $r \geqq 1$.
\end{abstract}

\section{Introduction}

Let $\theta$ be a continuous automorphism of a compact abelian group $G$. The classical automorphism theorem of Halmos [4] states that $\theta$ is strongly mixing with respect to the normalized Haar measure on $G$ if and only if it is ergodic. To explain our generalization, say $\theta$ is completely mixing in $G$ if and only if the following holds:

Given any integer $r \geqq 1$, any $r+1$ measurable subsets $A_{0}, \ldots, A_{r}$ of $G$, and any $r$ sequences $\left\{k_{i n}\right\}$ of positive integers such that $\lim _{n \rightarrow \infty} k_{\imath n}=\infty$ for all $1 \leqq i \leqq r$, we have

$$
\lim _{n \rightarrow \infty} \mu\left(A_{0} \cap \theta^{-k_{1 n}}\left(A_{1}\right) \cap \cdots \cap \theta^{-k_{r n}}\left(A_{n}\right)\right)=\prod_{j=0}^{r} \mu\left(A_{j}\right) .
$$

In an arbitrary space the above property may well be stronger than the condition of $r$-mixing introduced by Rohlin [9], since the latter involves the extra assumption that $\lim _{n \rightarrow \infty} \min _{i \neq j}\left|k_{i n}-k_{j n}\right|=\infty$. We prove the following generalization of the above result.

Theorem 1 (Endomorphism Theorem). Let $G$ be a compact abelian group with normalized Haar measure $\mu$, and let $\theta$ be a continuous surjective endomorphism of $G$. Then $\theta$ is $\mu$-invariant. Moreover, the following are equivalent:

(i) $\theta$ is completely mixing.

(ii) $\theta$ is r-mixing for all $r \geqq 1$.

* This work was supported in part by grants from NSERC 
(iii) $\theta$ is ergodic.

(iv) The induced homomorphism $\hat{\theta}$ has no non-trivial finite orbits on the character group $\hat{G}$.

(v) For every $n \geqq 1$ the endomorphism $I-\theta^{n}$ of $G$ is surjective, where I denotes the identity map on $G$.

Rohlin [9] proves the equivalence of (ii), (iv) above by a different method. Note also that the bilateral shift endomorphism is completely mixing but not exact, so the conclusion of Theorem 1 cannot be much strengthened.

Our interest in the dynamics of endomorphisms arises from the study of generalized automata. Let $G=\mathbb{Z}_{2}^{S}=\prod_{i \in S} \mathbb{Z}_{2}$, where $S$ is either $\mathbb{N}$ or $\mathbb{Z}$, and let $\theta$ be a continuous endomorphism of $G$. Then for each $i \in S$ there exists a finite subset $K_{i} \subset S$ such that $\theta(a)_{i}=\sum_{j \in K_{i}} a_{j}$ for all $a \in G$. Let $\rho(i)=\max \left\{j \in K_{i}\right\}$ and $\lambda(i)=$ $\min \left\{j \in K_{i}\right\}$. Assume that $\rho$ (and $\lambda$ in the case $S=\mathbb{Z}$ ) is strictly increasing and has no fixed points. Then Theorems 2 and 3 combine to say that in this case $\theta$ is onto, completely mixing, has periodic points of all orders, and the set of periodic points of $\theta$ is dense in $G$. Furthermore, Theorem 4 states that in the case $G=\mathbb{Z}_{2}^{\mathbb{N}}$ the above maps are also strongly transitive (i.e. $\bigcup_{n=0}^{\infty} \theta^{n}(V)=G$ for every non-empty open subset $V$ of $G$ ). As an immediate consequence of the above we have

Corollary 2. One-dimensional cellular automata, and the accumulator automata [1] are completely mixing.

The corollary may shed some light on the unpredictable space-time patterns produced by certain local automata. For example, some of Wolfram's class III automata [11] are clearly completely mixing. The corollary also strengthens a result due to Lind [6]. The accumulator automata $C: \mathbb{Z}_{2}^{\mathbb{N}} \rightarrow \mathbb{Z}_{2}^{\mathbb{N}}$ given by $(C x)_{i}=$ $x_{i+1}+\sum_{j=0}^{i} C_{i j} x_{j}$ were introduced in [1], where the algebraic structure of the set of periodic points associated with them were elucidated. The particular example of the twisted shift map $(T x)_{i}=x_{0}+x_{i+1}$ was utilized in [12] as a symbolic dynamical system in the enumeration of the bifurcations of the stable periodic cycles associated with quadratic maps of the interval.

The hypothesis that $\rho$ (and $\lambda$ ) in Theorems 2 and 3 be order preserving may well be too strong, as is shown by the following:

Theorem 5. Let $G=\mathbb{Z}_{2}^{S}$, and let $\theta: G \rightarrow G$ be defined by $\theta(x)_{i}=x_{\rho(i)}$, where $\rho: S \rightarrow S$ is injective and has no periodic points on $S$. Then $\theta$ is continuous, surjéctive, completely mixing, has points of all periods, and its set of periodic points is dense in $G$.

Whether Theorems 2 and 3 can still be proved under the weakened hypothesis of Theorem 5 remains to be seen.

\section{The Endomorphism Theorem}

Let $G$ be a compact abelian group (written additively) and let $\mu$ denote the normalized Haar measure on $G$. Let $T$ be a continuous $\mu$-invariant map of $G$ into 
itself. If $T$ is completely mixing then it is in particular strongly mixing (the case $r=1$ of $r$-mixing). The latter property has many interesting consequences, among which are ergodicity, sensitive dependence to initial conditions (at all points of $G$ ), and the fact that the set of periodic points of $T$ has measure zero in $G$ (unless some iterate of $T$ is the identity mapping on $G$ ). It is therefore of interest to determine conditions under which (algebraic) homomorphisms of $G$ are completely mixing. Before stating these conditions, recall that the character group $\hat{G}$ of $G$ consists of all continuous homomorphisms $\lambda$ of $G$ into the group of complex numbers of modulus one. Given a continuous endomorphism $\theta$ of $G$, the induced homomorphism $\hat{\theta}$ on $\hat{G}$ is defined by $\hat{\theta}(\lambda)(x)=\lambda(\theta(x))$ for all $x \in G$. We also write $1_{G}$ for the trivial character on $G$ (i.e. $1_{G}(x)=1$ for all $x \in G$ ).

Proof of Theorem 1. The $\mu$-invariance of $\theta$ is well-known ([8], I.7.2). The equivalence of (iv) and (v) is also easy to see. For, given any $n \geqq 1$, the set $H=\left\{x-\theta^{n}(x)\right.$ : $x \in G\}$ is in fact a subgroup of $G$, and $\hat{\theta}^{n}(\lambda)=\lambda$ if and only if the restriction of $\lambda$ to $H$ is the trivial character $1_{H}$. Moreover if $H \neq G$ then any non-trivial character of $G / H$ gives rise to a character of $G$ which is trivial on $H$. Thus $H \neq G$ is equivalent to the existence of non-trivial character $\lambda$ of $G$ such that $\hat{\theta}^{n}(\lambda)=\lambda$. This establishes the equivalence of (iv) and (v).

The implications (i) $\Rightarrow$ (ii) $\Rightarrow$ (iii) are trivial. The proof that (iii) implies (iv) is essentially Halmos' observation [3] that if $\hat{\theta}^{n}(\lambda)=\lambda$ and $\lambda \neq 1_{G}$, then $f=$ $\sum_{i=0}^{n-1} \hat{\theta}^{i}(\lambda)$ is a non-constant function in $\mathscr{L}^{2}(G)$ such that $f \theta=f$. This implies that $\theta$ is not ergodic ([8], II.2.1).

It remains to prove that (iv) implies (i). Define the linear operator $U_{\theta}=U$ on $\mathscr{L}^{2}(G)$ by $U(f)=f \theta$ for all $f \in \mathscr{L}^{2}(G)$. Consider the equation

$$
\lim _{n \rightarrow \infty}\left\langle f_{0} U^{k_{1 n}}\left(f_{1}\right) \cdots U^{k_{r n}}\left(f_{r}\right), 1_{G}\right\rangle=\prod_{j=0}^{r}\left\langle f_{j}, 1_{G}\right\rangle
$$

where $f_{0}, \ldots, f_{r} \in \mathscr{L}^{2}(G)$. Clearly $\theta$ is completely mixing if (1) holds when the $f_{i}$ are the characteristic functions of the sets $A_{i}$. We claim that (1) is in fact true for all $f_{i} \in \mathscr{L}^{2}(G)$. It is sufficient to show that (1) holds when the $f_{i}$ are linear combinations of characters, for then the fact that $\hat{G}$ is dense in $\mathscr{L}^{2}(G)([7], 38 \mathrm{D})$, together with some straightforward estimates, show that (1) holds for all $f_{i} \in \mathscr{L}^{2}(G)$.

Note that if $\lambda$ is a character then $U^{n}(\lambda)=\lambda \theta^{n}=\hat{\theta}^{n}(\lambda)$. Let $f_{i}=\sum_{j=0}^{n} c_{i j} \lambda_{j}$, where $\lambda_{0}=1_{G}$, the $c_{i j} \in \mathbb{C}$, and the $\lambda \hat{j} \in \hat{G}$. Then the left-hand side of (1) consists, apart from the term $c_{00} c_{10} \cdots c_{r 0}=\prod_{j=0}^{r}\left\langle f_{J}, 1_{G}\right\rangle$, of complex multiples of terms of the form $\left\langle\lambda_{i_{0}} \hat{\theta}^{k_{1 n}}\left(\lambda_{i_{1}}\right) \ldots \hat{\theta}^{k_{r n}}\left(\lambda_{i_{r}}\right), 1_{G}\right\rangle$, where $0 \leqq i_{0}, \ldots, i_{r} \leqq m$, and at least one $\lambda_{{ }^{\prime} S} \neq 1_{G}$. An application of (the multiplicative version of) the $H-S$ Lemma (cf. Sect. 5) shows that for all sufficiently large $n, \lambda_{i_{0}} \hat{\theta}^{k_{1 n}}\left(\lambda_{i_{1}}\right) \cdots \hat{\theta}^{k_{r n}}\left(\hat{\lambda}_{i_{r}}\right) \neq 1_{G}$, whence the above inner product is zero. This establishes (1) for all linear combinations of characters, and hence concludes the proof of the theorem.

In particular using (iv) of Theorem 1 we have 
Corollary 1. Let $\theta$ be a continuous surjective endomorphism of a compact abelian group. Then the ergodicity of any power of $\theta$ implies the ergodicity of all powers of $\theta$.

The above is of course false for arbitrary ergodic maps $T$; more precisely, the ergodicity of $T$ does not imply that of any of its iterates.

\section{Complete Mixing and Strong Transitivity in $\mathbb{Z}_{2}^{S}$}

Henceforth we confine ourselves to the case where $G$ is a binary sequence space. To treat infinite and bi-finite sequences simultaneously we write $G=\mathbb{Z}_{2}^{S}=\prod_{i \in S} \mathbb{Z}_{2}$, where $S$ is either $\mathbb{N}=\{0,1, \ldots\}$ or $\mathbb{Z}$. In what follows $\theta$ denotes a continuous endomorphism of $G$.

It is easy to see that for each $i \in S$ there exists a finite subset $K_{i} \subseteq S$ such that $\theta(a)_{i}=\sum_{j \in K_{i}} a_{j}$ for all $a \in G$. Conversely, any choice of finite subsets $K_{i}$ of $S$ gives rise to a continuous homomorphism of $G$ defined as in the above formula. Therefore, the dynamical properties of $\theta$ are determined solely by the collection of subsets $\left\{K_{i}: i \in S\right\}$. We begin with some elementary results.

Lemma 1. Let $G=\mathbb{Z}_{2}^{S}$, and let $\theta$ be a continuous endomorphism of $G$. For any $n \geqq 1$ and any $i \in S$ write the $i$-th component of $\theta^{n}$ in the form

$$
\theta^{n}(x)_{i}=\sum_{j \in K(n, i)} x_{j}, \quad \text { all } x \in G .
$$

Then for $n \geqq 2$ the sets $K(n, i)$ are defined recursively by

$$
K(n, i)=\Delta_{j \in K(n-1, i)} K(1, j) .
$$

$(A \Delta B=(A \backslash B) \cup(B \backslash A)$ is the symmetric difference of the sets $A$ and $B$.

Proof. By definition $\sum_{j \in K(n, i)} x_{j}=\theta^{n}(x)_{i}=\theta^{n-1}(\theta(x))_{i}=\sum_{j \in K(n-1, i)} \theta(x)_{j}=\sum_{j \in K(n-1, i)}$ $\sum_{r \in K(1, j)} x_{r}$. The only terms $x_{r}$ that survive in the last double sum are those for which $r$ occurs an odd number of times in the various $K(1, j)$ for $j \in K(n-1, i)$. But this is merely another way of describing the symmetric difference of the $K(1, j)$.

For example if $K(1, i)=\{0, i+1\}$ for all $i \in \mathbb{N}$, then $K(2, i)=K(1,0) \Delta K(1, i+1)=$ $\{0,1\} \Delta\{0, i+2\}=\{1, i+2\}$, and more generally $K(n, i)=\{n-1, n+i\}$.

Lemma 2. In the notation of Lemma 1 assume that $\theta$ is surjective, and that $K(1, i) \subseteq$ $\{-i, \ldots,+i\}$ for all $i \in S$. Then $\theta$ is not ergodic.

Proof. A trivial inductive argument shows that $K(n, i) \subseteq\{-i, \ldots, i\}$ for all $i$ and $n$, and so $K(n, i)=K(m, i)$ for some $m>n$. In view of the definitions this means that $\theta^{n}(x)_{i}=\theta^{m+n}(x)_{i}$ for all $x \in G$, and since $\theta^{n}$ is surjective we get $y_{i}=\theta^{m}(y)_{i}$ for all $y \in G$. But then the $i$-th component of $I-\theta^{m}$ is identically zero, and the conclusion follows from Theorem 1.

It is apparent from Lemma 2 that the maximum and minimum elements of 
the sets $K(n, i)$ exert considerable influence over the dynamical properties of $\theta$. Let

$$
\begin{aligned}
& \rho(i)=\max \{j: j \in K(1, i)\}, \\
& \lambda(i)=\min \{j: j \in K(1, i)\},
\end{aligned}
$$

for all $i \in S$. A case in which the ergodicity of $\theta$ can be asserted is when $\rho$ (and $\lambda$ when $S=\mathbb{Z}$ ) are both strictly increasing, i.e. $i<j$ implies that $\rho(i)<\rho(j)$.

Theorem 2. Let $G=\mathbb{Z}_{2}^{S}$ and let $\theta$ be a continuous endomorphism of $G$. Assume that $\rho$ (and $\lambda$ in the case $S=\mathbb{Z}$ ) is strictly increasing and has no fixed points. Then $\theta$ is surjective and completely mixing.

Proof. The following properties of the map $\rho$ are trivial to verify: $\rho$ is injective; $\rho^{n}(i) \neq i$ for all $n \geqq 1$ and all $i \in S$; if $j<\rho^{n}(j)$ for some $j \in S$ then $i<\rho^{n}(i)$ for all $i \geqq j$; if $\rho^{n}(j)<j$ for some $j \in S$ then $\rho^{n}(i)<i$ for all $i \leqq j$.

We treat the cases $S=\mathbb{N}$ and $S=\mathbb{Z}$ separately, beginning with the former. Here $0<\rho^{n}(0)$, and so $i<\rho^{n}(i)$ for all $i \in S$. We first show that $\theta$ is surjective. This means that given $a \in G$ we have to solve the system of equations

$$
\sum_{j \in K(1, i)} x_{j}=a_{i}, \text { all } i \in S,
$$

for some $x \in G$. The solution may be found as follows: since $0<\rho(0)<\rho(1)<$ $\rho(2)<\cdots$, we begin by defining $x_{r}$ arbitrarily for $0 \leqq r<\rho(0)$, use (1) to find $x_{\rho(0)}$, define $x_{r}$ arbitrarily for $\rho(0)<r<\rho(1)$, use (1) to find $x_{\rho(1)}$, and so on.

The surjectivity of $I-\theta^{n}$ is just as easy. Given $a \in G$ we have to find $x \in G$ satisfying

$$
x_{i}-\sum_{j \in K(n, i)} x_{j}=a_{i}, \quad \text { all } i \in S .
$$

By induction on $n$, the maximum element of $K(n, i)$ is $\rho^{n}(i)>i$. We may thus solve (2) exactly as we did (1), since (2) determines each $x_{\rho^{n}(i)}$ in terms of the $x_{j}$ with $j<\rho^{n}(i)$. The ergodicity of $\theta$ now follows from Theorem 1 .

In the case $S=\mathbb{Z}$ we still have to solve the systems (1) and (2) for any given $a \in G$. We indicate the solution of the more complicated system (2) only. Let $n \geqq 1$ be fixed.

Suppose first that $i<\lambda^{n}(i) \leqq \rho^{n}(i)$ for all $i \in S$. Then the left-hand side of (2) has the unique maximal subscript $\rho^{n}(i)$ and the unique minimal subscript $i$ for all $i \in S$. Begin by defining the $x_{r}$ arbitrarily for $0 \leqq r<\rho^{n}(0)$. For $i>0$, use (2) to define $x_{\rho^{n}(i)}$ in terms of previously defined components (some of which may be arbitrary), and for $i<0$, use (2) to define $x_{i}$ (with no arbitrary choices).

The case where $\lambda^{n}(i) \leqq \rho^{n}(i)<i$ for all $i \in S$ can be treated analogously. We are left with the possibility that $i<\lambda^{n}(i) \leqq \rho^{n}(i)$ for some $i \in S$, and $\lambda^{n}(j) \leqq \rho^{n}(j)<j$ for some $j \in S$. It is then easy to see that there exist integers $j_{1}$ and $j_{2}$ such that $i<\lambda^{n}(i) \leqq \rho^{n}(i)$ for all $i \geqq j_{1}$, and $\lambda^{n}(i) \leqq \rho^{n}(i)<i$ for all $i \leqq j_{2}$. Moreover $j_{2}=j_{1}$, or $j_{2}=j_{1}-1$. We thus have $\cdots<\lambda^{n}\left(j_{2}-1\right)<\lambda^{n}\left(j_{2}\right)<j_{2} \leqq j_{1}<\rho^{n}\left(j_{1}\right)<\rho^{n}\left(j_{1}+1\right)<\cdots$ Define $x_{r}$ arbitrarily for $\lambda^{n}\left(j_{2}\right)<r<\rho^{n}\left(j_{1}\right)$. For $i \geqq j_{1}$ the term $\rho^{n}(i)$ is the highest subscript occurring in (2), while for $i \leqq j_{2}$ the term $\lambda^{n}(i)$ is the lowest subscript occurring in (2). Therefore the system can be solved consistently, showing that $I-\theta^{n}$ is surjective. Similarly (1) may be solved along the same lines as above. The result follows. 
Corollary 2. One-dimensional linear cellular automata, and the general accumulator maps of [1] are completely mixing.

Proof. In the case of cellular automata $\rho(i)=i+r_{1}$ and $\lambda(i)=i+r_{2}$ where $r_{1}$ and $r_{2}$ are fixed non-zero integers. The accumulator maps are defined on $S=\mathbb{N}$ and have $\rho(i)=i+1$. In each case the conditions of Theorem 2 are trivially verified.

The above considerably strengthens Lind's result [6] for the map $\theta(x)_{i}=$ $x_{i-1}+x_{i+1}$. The class III cellular automaton with code number 42 ([11], page $7)$ is the case $\theta(x)_{i}=x_{i-2}+x_{i-1}+x_{i}+x_{i+1}+x_{i+2}$ of Corollary 2.

Write $P_{n}=\left\{x \in G: \theta^{n}(x)=x\right\}$ for $n \geqq 1$. If $\theta$ is a linear map then each $P_{n}$ is a subspace of $G$ (regarded as a vector space over the field of two elements). In particular the cardinality of $P_{n}$ is either a finite power of 2 or that of the continuum. As has already been remarked, the fact that the maps $\theta$ of Theorem 2 are strongly mixing implies that $P(G)=\bigcup_{n=0}^{\infty} P_{n}$ has measure zero in $G$. There are, nonetheless, many periodic points.

Theorem 3. Let $G=\mathbb{Z}_{2}^{S}$ and let $\theta$ be a continuous endomorphism of $G$. Assume that $\rho$ (and $\lambda$ in the case $S=\mathbb{Z}$ ) is strictly increasing and has no fixed points. Then $\theta$ has periodic points of all orders, and the set of periodic points of $\theta$ is dense in $G$.

Proof. Clearly we have $\theta^{n}(x)=x$ if and only if $\left(I-\theta^{n}\right)(x)=0$. To show the existence of points of period exactly $n$ we need a closer look at the proof of the surjectivity of the maps $I-\theta^{n}$ given in Theorem 2. For example in the case $S=\mathbb{N}$ a point of period dividing $n$ is a solution of $x_{i}+\sum_{j \in K(n, i)} x_{j}=0$ for all $i \in S$. In particular if $d$ is a proper divisor of $n$ then $x_{\rho^{d}(0)}$ is uniquely determined by the $x_{j}, 0 \leqq j<\rho^{d}(0)$ (which have been chosen arbitrarily). However for any choice of the components $a_{j}, 0 \leqq j<\rho^{d}(0)$, we can find $x, y \in P_{n}$ with $x_{j}=y_{j}=a_{j}$ for $0 \leqq j<\rho^{d}(0), x_{\rho^{d}(0)}=0$, and $y_{\rho^{d}(0)}=1$, so at most one of $x$ or $y$ can have period $d$. This plainly establishes the existence of points of period exactly $n$. Similarly given any $m \geqq 1$ there exists $n$ such that $\rho^{n}(0)>m$. Thus given any $a \in G$ one can find $x \in P_{n}$ such that $x_{i}=a_{i}$ for $0 \leqq i \leqq m<\rho^{n}(0)$. This proves that the set of periodic points is dense in $G$. The proof of the (entirely analogous) case $S=\mathbb{Z}$ is left to the reader.

A well-known consequence of the ergodicity of the maps $\theta$ is that they are all topologically transitive, i.e., for any non-empty open set $V$, the set $\bigcup_{n=0}^{\infty} \theta^{n}(V)$ is dense in $G$. Following [2] we say $\theta$ is strongly transitive if $\bigcup_{n=0}^{\infty} \theta^{n}(V)=G$ for all non-empty open subsets $V$ of $G$. In this direction we have

Theorem 4. Let $G=\mathbb{Z}_{2}^{\mathbb{N}}$, and let $\theta$ be a continuous endomorphism of $G$ such that $\rho$ is strictly increasing and has no fixed points. Then $\theta$ is strongly transitive.

Proof. We have to prove that given any $a, b \in G$ (possibly $a=b$ ) and any neighbourhood $V$ of $a$, there exists an integer $m$ and an element $x \in V$ such that $\theta^{m}(x)=b$. A fundamental system of open neighbourhoods of the identity is given by the 
cylinder sets $V_{n}=\left\{x \in G: x_{i}=0\right.$ for $\left.0 \leqq i \leqq n\right\}$ for all $n \geqq 0$. Thus given $a, b \in G$ and $n \geqq 0$ we have to find $m \geqq 1$ and $x \in G$ such that $\theta^{m}(x)=b$ and $x_{i}=a_{i}$ for $0 \leqq i \leqq n$. Choose any $m$ such that $\rho^{m}(0)>n$. Set $x_{i}=a_{i}$ for $0 \leqq i \leqq n$, and use the equations $\sum_{j \in K(m, i)} x_{j}=b_{i}$ to determine the $x_{\rho^{m}(i)}$ for all $i \geqq 0$ (other components of $x$ may be chosen at will).

The result, of course, does not extend to the case $S=\mathbb{Z}$. Indeed, even the bi-infinite shift map $\sigma(x)_{i}=x_{i+1}$ does not possess strong transitivity, since from almost no neighbourhood of $\overline{1}$ (all 1's) can one reach $\overline{10}$ (alternate 1's and 0's) under any iterate of $\sigma$.

\section{Other Examples}

Unfortunately the condition that $\rho$ (and $\lambda$ ) be strictly increasing is not always necessary for the truth of Theorems 2 and 3, as the following result shows

Theorem 5. Let $G=\mathbb{Z}_{2}^{S}$, and let $\theta: G \rightarrow G$ be defined by $\theta(x)_{i}=x_{\rho(i)}$, where $\rho$ is injective and has no periodic points on $S$. Then $\theta$ is continuous, surjective, completely mixing, has points of all periods, and the set of periodic points of $\theta$ is dense in $G$.

Proof. The surjectivity of $\theta$ is trivial since given $a \in G$ one simply defines $x \in G$ by $x_{\rho(i)}=a_{i}$ for all $i \in S$, and $x_{j}$ is arbitrary if $j \notin \rho(\mathrm{S})$. The definition is consistent since $\rho$ is injective.

Clearly $K(n, i)=\left\{\rho^{n}(i)\right\}$, so surjectivity of $I-\theta^{n}$ amounts to the solution, for $x$, of the system

$$
x_{i}-x_{\rho^{n}(i)}=a_{i}, \quad \text { all } \quad i \in S,
$$

where $a \in G$ is given. Let $n$ be fixed, and define an equivalence relation $\sim_{n}$ on $S$ by: $i \sim{ }_{n} j$ if either $i=\rho^{r n}(j)$ or $j=\rho^{r n}(i)$ for some $r \geqq 0$ (the transitivity of $\sim$ depends upon the injectivity of $\rho$ ). Consider an equivalence class $\left[i_{0}\right]_{n}$, where $i_{0} \in S$. Then the relation $\prec$, where $j \prec k$ if and only if $j=\rho^{n}(k)$, induces a strict total order on $\left[i_{0}\right]_{n}$ (anti-symmetry follows from the fact that $\rho$ has no periodic points, so $j=\rho^{n}(k)$ and $k=\rho^{n}(j)$ cannot hold simultaneously). We may therefore write $\left[i_{0}\right]_{n}=$ $\left\{\ldots, i_{-2}, i_{-1}, i_{0}, i_{1}, i_{2}, \ldots\right\}$, where $\rho^{n}\left(i_{k}\right)=i_{k+1}$ for all integers $k$. The subsystem of (1) indexed by the elements of $\left[i_{0}\right]_{n}$ thus has the form

$$
\begin{gathered}
x_{i_{-1}}-x_{i_{0}}=a_{i_{-1}} \\
x_{i_{0}}-x_{i_{1}}=a_{i_{0}}
\end{gathered}
$$

One may choose $x_{i_{0}}$ arbitrarily, and solve the system by forward/backward substitution. Of course the subsystems indexed by different equivalence classes are disjoint, so the above method of solution is consistent. This establishes the ergodicity of $\theta$.

The existence of periodic points is the case $a=0$ of the above construction, 
and is clearly equivalent to the conditions $x_{r}=x_{i}$ for all $r \in[i]_{n}$. To show the existence of points of period exactly $n$ we need merely observe that if $d$ is a proper divisor of $n$ then $[i]_{n}$ is a proper subset of $[i]_{d}$ (for example $\rho^{d}(i) \in[i]_{d} \backslash[i]_{n}$ ). Consider the $n$ distinct equivalence classes $[i]_{n},[\rho(i)]_{n}, \ldots,\left[\rho^{n-1}(i)\right]_{n}$. By assigning values of 0 or 1 to the components of $x$ on the above classes (with an appropriate definition elsewhere) we obtain $2^{n}$ vectors $x$ satisfying $\theta^{n}(x)=x$. If $d$ is a divisor of $n$ then $\sim_{d}$ partitions $\left\{i, \rho(i), \ldots, \rho^{n-1}(i)\right\}$ into $d$ equivalence classes, whereby one obtains only $2^{d}$ points whose period divides $d$. But $\sum_{\substack{d \mid n \\ d \neq n}} 2^{d} \leqq \sum_{i=1}^{n-1} 2^{i}=2^{n}-2$, so there is at least one point whose period is exactly $n$.

To show that the set of periodic points is dense in $G$, let $J$ be any finite subset of $S$. If $i \in J$ then $\rho^{n}(i) \notin J$ for all sufficiently large $n$ (for if $\rho^{n}(i) \in J$ for an infinity of values of $n$ then $\rho^{n}(i)=\rho^{m}(i)$ for some $n \neq m$, which is impossible). Since $J$ is finite it follows that $J \cap \rho^{n}(J)=\varnothing$ for all sufficiently large $n$. This means that the classes $[i]_{n}$, for $i \in J$, are all distinct, and so the components of $x$ indexed by them can be assigned arbitrary values. In other words, given any $y \in G$ and any finite $J$ there exists $x \in P_{n}$ such that $x_{i}=y_{i}$ for all $i \in J$, as required.

Observe that the conditions on $\rho$ in the above theorem cannot be relaxed. For example if $\rho(i)=\rho(j)$ for some $i \neq j$ then $(\theta x)_{i}=(\theta x)_{j}$ for all $x \in G$, so $\theta$ is not surjective, while if $\rho^{n}(i)=i$ for some $n \geqq 1$ then $I-\theta^{n}$ is not surjective.

Finally, we observe that the maps in Theorem 5 are not in general strongly transitive, even when $S=\mathbb{N}$. For example consider the map $\rho$ on $\mathbb{N}$ given by $\cdots \rightarrow 8 \rightarrow 6 \rightarrow 4 \rightarrow 2 \rightarrow 0 \rightarrow 1 \rightarrow 3 \rightarrow 5 \rightarrow \cdots$ Let $b_{i}=1$ for all $i \in \mathbb{N}$. Then no $x \in G$ such that $x_{0}=0$ can map to $b$ under any iterate of $\theta$, for given any $n$ we have $\theta^{n}(x)_{2 n}=$ $x_{\rho^{n}(2 n)}=x_{0}=0 \neq b_{2 n}$. Hence from almost no neighbourhood of the identity can one reach $b$. The problem of relaxing the conditions on $\rho$ (and $\lambda$ ) in Theorem 2 and 3 still remains.

\section{The Homomorphism-Separation Lemma}

Here we prove the following purely algebraic result, needed for the proof of Theorem 1:

The H-S Lemma. Let $M$ be an additive abelian group, and let $\psi$ be an endomorphism of $M$ such that

$$
\psi \text {, and } 1-\psi^{n} \text { for all } n \geqq 1 \text {, are } 1-1 \text { maps of } M \text {. }
$$

Let $\left\{k_{1 n}\right\}, \ldots,\left\{k_{r n}\right\}$ be sequences of positive integers satisfying $\lim _{n \rightarrow \infty} k_{i n}=\infty$ for $1 \leqq i \leqq r$. If $x_{0}, \ldots, x_{r}$ are elements of $M$ such that

$$
x_{0}=\sum_{i=1}^{r} \psi^{k_{n n}}\left(x_{i}\right), \text { for all } n,
$$

then $x_{0}=\cdots=x_{r}$.

Proof. The subring $R=\mathbb{Z}[\psi]$ of End $(M)$ generated by $\psi$ is Noetherian. Replacing $M$ by its $R$-submodule generated by the $x_{i}$ we may assume that $M$ is a finitely 
generated left $R$-module. Also observe that if $M$ is the direct sum $M_{1} \oplus \cdots \oplus M_{k}$ of its $R$-submodules $M_{i}$ and if each $x_{i}=\sum_{j=1}^{k} y_{i j}$, where the $y_{i j} \in M_{j}$, then (2) its equivalent to the $k$ systems $y_{0 j}=\sum_{i=1}^{r} \psi^{k_{i n}}\left(y_{i j}\right)=0,1 \leqq j \leqq k$, and also the restriction of $\psi$ to each $M_{j}$ also satisfies (1). Since $x_{0}=\cdots=x_{r}=0$ if and only if all the $y_{i j}=0$, it is sufficient to assume from the beginning that all the $x_{i} \in M_{j}$.

We prove the lemma in a series of steps.

1. Consider the special case where $M$ has a countable basis $u_{1}, u_{2}, \ldots$ over some field $F$, and $\psi$ is the $F$-linear one-sided shift map $\psi\left(u_{i}\right)=u_{i+1}$ for all $i$. Clearly for all sufficiently large $n$ the right-hand side of (2) cannot contain any of the $u_{i}$ involved in $x_{0}$. This implies that $x_{0}=0$, and simple induction on $r$ concludes the proof in this case.

2. Now consider the case where char $R=0, M$ has no $R$-torsion (i.e. if $0 \neq x \in M$ and $0 \neq r \in R$ then $r x \neq 0$ ), and let $S=R \otimes_{\mathbb{Z}} \mathbb{Q}$. Then $N=M \otimes_{\mathbb{Z}} \mathbb{Q}$ has no $S$-torsion, and $\psi$ extends naturally to an endomorphism, still denoted by $\psi$, of $N$. Since $S$ is a principal ideal domain ([5], Theorem 2.15) we can write $N=S y_{1} \oplus \ldots$ $\oplus S y_{m}$, where each $S y_{i}$ is a free cyclic $S$-module ([5], Theorem 3.10). Consider one of the summand, $S y_{j}$ say. Since this module has no $S$-torsion it follows that $y_{j}, \psi\left(y_{j}\right)$, $\psi^{2}\left(y_{j}\right), \ldots$ form a $\mathbb{Q}$-basis of $S y_{j}$, and clearly $\psi$ acts as the shift map relative to this basis. An application of Step 1. concludes this case.

3. Now assume merely that $M$ has no $\mathbb{Z}$-torsion (i.e. if $0 \neq x \in M$ and $n>0$ is an integer then $n x \neq 0$ ). As in Step 2. Let $N=M \otimes_{\mathbb{Z}} \mathbb{Q}$ and $S=R \otimes_{\mathbb{Z}} \mathbb{Q}$. Then $N$ is a direct sum of free cyclic modules of the form $S y$, and the $R$-torsion submodule $T$ of $N$. In view of Step 2 we may assume that $x_{0}, \ldots, x_{r} \in T$, and that in fact they generate $T$ as $S$-module.

Since $S$ is a principal ideal domain and $T$ is finitely $S$-generated, there exists a non-zero polynomial $g(\psi) \in \mathbb{Q}[\psi]$ such that $g(\psi) x=0$ for all $x \in T$, whence $g(\psi)=0$. Thus for some integer $n$ and rational numbers $q_{i}$ we have $\psi^{n}=\sum_{0 \leqq i<n} q_{i} \psi^{i}$, and therefore $T$ has finite $\mathbb{Q}$-dimension. In particular the injective maps $\underset{0}{0 \leq n}$ and $1-\psi^{n}$ are invertible. Put $V=T \otimes_{\mathbb{Q}} F$, where $F$ denotes the algebraic closure of $\mathbb{Q}$ in $\mathbb{C}$, and consider an $\psi$-composition series $\{0\}=V_{0} \subset V_{1} \subset \cdots \subset V_{m}=V$ of $V$. Since the map induced by $\psi$ on each factor $V_{i} / V_{i-1}$ also satisfies (1) we may assume (by induction on $\operatorname{dim}_{F} V$ ) that $V=V_{1}$. But now the absolute irreducibility of the action of $\psi$ on $V$ (and Schur's Lemma) implies that $\operatorname{dim}_{F} V=1$, say $V=F u$. We have $x_{i}=\lambda_{i} u$ and $\psi(u)=\alpha u$, where the $\lambda_{i}$ and $\alpha$ belong to $F$. By (1) we know that $\alpha$ is not a root of unity. Clearly (2) is equivalent to $\lambda_{0}=\sum_{i=1}^{r} \lambda_{i} \alpha^{k_{1 n}}$ for all $n$, which implies that $\lambda_{0}=0$. (For example it is well-known ([10], IV.3, Theorem 8) that one can find a valuation $\|$ on the algebraic number field $\mathbb{Q}\left(\lambda_{0}, \ldots, \lambda_{r}, \alpha\right)$ such that $|\alpha|<1$, whence $\lambda_{0}=0$ follows upon letting $n \rightarrow \infty$.)

4. Now consider the case where $M$ is a torsion group. Then we can write $M=\oplus M_{p}$, where the sum ranges over all primes $p$ and $M_{p}=\left\{x \in M: p^{e} x=0\right.$ for some $\left.e \geqq 0\right\}$. Since only a finite number of the $M_{p}$ can be non-zero we may assume that $M=M_{p}$.

Consider first the case where $p x=0$ for all $x \in M$. Then $M$ is a finitely generated 
module over $R^{\prime}=\mathbb{Z}_{p}[\psi]$. Since $R^{\prime}$ is a principal ideal domain we have $M=$ $R^{\prime} y_{1} \oplus \cdots \oplus R^{\prime} y_{m} \oplus T$, where the $R^{\prime} y_{i}$ are free cyclic $R^{\prime}$-modules and $T$ is the $R^{\prime}$ torsion submodule of $M$. Observe that $T=\{0\}$, for if $x \in T$ then by definition $g(\psi) x=0$ for some $0 \neq g(\psi) \in \mathbb{Z}_{p}[\psi]$, whence $\left(1-\psi^{n}\right) x=0$ for some $n \geqq 1$. In view of (1) this implies $x=0$, as claimed. As in Step 2 the action of $\psi$ on each $R^{\prime} y_{j}$ is like the shift map, and so the conclusion $x_{i}=0$ follows from Step 1 .

In general there exists $e \geqq 1$ such that $p^{e} x=0$ for all $x \in M$. As $M$ is a torsion group the map induced by $\psi$ on each $R^{\prime}$-module $p^{i} M / p^{i+1} M$ also satisfies (1). By the previous paragraph we find that $x_{0}, \ldots, x_{r}$ belong to every $p^{i} M$, and in particular to $p^{e} M=\{0\}$, as required.

5. Finally in the case of an arbitrary group $M$ let $T=\{x \in M: n x=0$ for some $n>0\}$. Then $T$ is a submodule of $M$, and the map induced by $\psi$ on the $\mathbb{Z}$-torsion-free module $M / T$ satisfies (1). An application of Step 3 to $M / T$ shows that all the $x_{i} \in T$, and proof is concluded by an application of Step 4 to $T$.

\section{References}

1. Allison, B. N., Pounder, J. R., Rogers, T. D.: Algebraic structure of automata with spatial memory. University of Alberta preprint (August 1987)

2. Coven, E. M., Mulvey, I.: Transitivity and the centre for maps of the circle. Ergod. Theory Dynam. Syst. 6, 1-8 (1986)

3. Halmos, P. R.: On automorphisms of compact groups. Bull AMS 49, 619-624 (1943)

4. Halmos, P. R.: Lectures on ergodic theory. New York: Chelsea 1956

5. Jacobson, N.: Basic algebra I. San Francisco: Freeman 1980

6. Lind, D. A.: Ergodic theory and cellular automata, in: Cellular automata. Proceedings of an Interdisciplinary Workshop. Farmer, D., Toffoli, T., Wolfram, S. (eds.). Amsterdam: North-Holland 1984

7. Loomis, L. H.: An introduction to abstract harmonic analysis. Princeton, NJ: Van Nostrand 1953

8. Mañé, R.: Ergodic theory and differentiable dynamics. Berlin, Heidelberg, New York: Springer 1987

9. Rohlin, V. A.: On endomorphisms of compact commutative groups. Izv. Akad. Nauk SSSR. Ser. Mat. 13, 329-340 (1949)

10. Weil, A.: Basic number theory. Berlin, Heidelberg, New York: Springer 1974

11. Wolfram, S. A.: Universality and complexity in cellular automata. In Cellular automata. Proceedings of an Interdisciplinary Workshop. Farmer, D., Toffoli, T., Wolfram, S. (eds.). Amsterdam: NorthHolland 1984

12. Weiss, A., Rogers, T. D.: The number of orientation reversing cycles in the quadratic map. Can. Math. Soc. Conf. Proc. 8 (1987)

Communicated by J.-P. Eckmann

Received January 19, 1988 